Unfallchirurg 2014 · 118:376-376

DOI 10.1007/s00113-014-2674-4

Online publiziert: 22. März 2015

c) Springer-Verlag Berlin Heidelberg 2014

A.P. Verheyden

Ortenau-Klinikum, Lahr-Ettenheim

\title{
Kommentar zu: Zervikale Myelopathie nach leichter HWS-Distorsion
}

notfallmäßig dekomprimiert werden. Liegt eine inkomplette Situation ohne Dynamik in den ersten 6-24 h vor, tendieren die meisten Wirbelsäulenchirurgen zum operativen Vorgehen.

$\mathrm{Zu}$ 2.: Eine Kortisongabe bei neurologischer Symptomatik nach Trauma ist nicht mehr indiziert, da zwischenzeitlich belegt werden konnte, dass die Nachteile eventuellen Vorteilen gegenüber klar überwiegen. Entsprechende Leitlinien der Fachgesellschaften liegen vor. Wird allerdings eine Dekompression durchgeführt, verwenden einige Operateure lokal oder systemisch Steroide unter der Vorstellung, das zusätzliche Trauma so eventuell minimieren zu können. Studien, die diesbezüglich eine Wirkung belegen, gibt es nicht.

$Z u$ 3.: Wesentlich ist eine ausreichende Dekompression, die in den meisten Fällen von ventral erfolgt. Bei speziellen Konfigurationen der spinalen Stenose mit v. a. dorsalen Einengungen ist aber auch ein rein dorsales oder gar kombiniertes Vorgehen indiziert. Ob beim ventralen Vorgehen mikrochirurgisch segmental vorgegangen wird oder längerstreckige Korporektomien mit anschließendem Wirbelkörperersatz durchgeführt werden, hängt von der Konfiguration der Stenose und bis zu einem gewissen Grad auch von der Vorliebe und Erfahrung des Operateurs ab. Da die vorgeschädigten neurologischen Strukturen maximale Stabilität benötigen, ist neben den Cages die Verwendung einer Platte zu empfehlen. Im vorliegenden Fall ist es ohne Platte offensichtlich gut gegangen, mit einer zusätzlichen Stabilisierung wäre es sicherer gewesen.
Den Autoren ist für die wichtige Falldarstellung zu danken, der die Problematik exemplarisch darstellt. Auch erfahrene Wirbelsäulenchirurgen kommen bei dieser Problematik immer wieder zum Nachdenken, welche Therapieoption beim jeweiligen Patienten die beste ist. Jeder Fall muss individuell abgewogen werden, zahlreiche weitere Faktoren, die hier in der Kürze nicht dargestellt werden können, müssen miteinbezogen werden. Um so wichtiger ist es, beim Leser die Sensibilität dafür zu schärfen, bei Patienten mit nicht eindeutiger neurologischer Symptomatik schnell zu reagieren und wenn im eigenen Haus keine hohe Expertise für solche Krankheitsbilder vorliegt, den $\mathrm{Pa}$ tienten sofort in ein entsprechend ausgewiesenes Zentrum zu verlegen.

\section{Korrespondenzadresse}

Prof. Dr. A.P. Verheyden

Ortenau-Klinikum

Klostenstr. 19, 77933 Lahr-Ettenheim

akhil.verheyden@le.ortenau-klinikum.de

\section{Einhaltung ethischer Richtlinien}

Interessenkonflikt. A.P. Verheyden gibt an, dass kein Interessenkonflikt besteht.

Dieser Beitrag beinhaltet keine Studien an Menschen oder Tieren. 